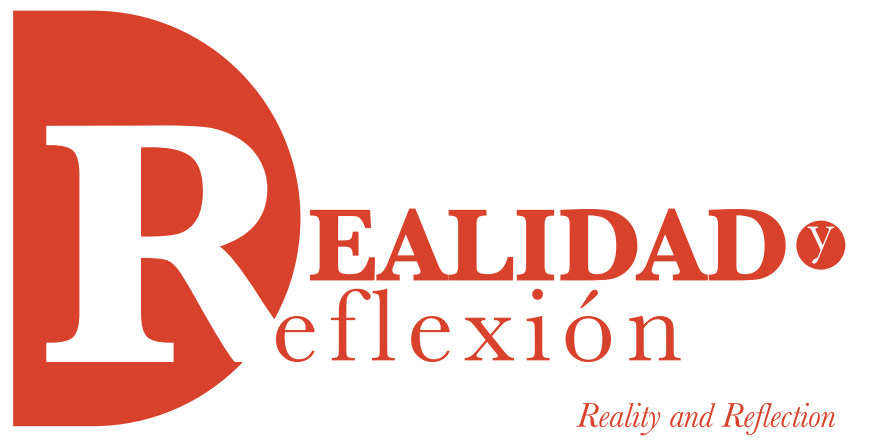

ISSN 1992-6510

e-ISSN 2520-92990

Año 17, N 45, San Salvador, El Salvador, Centroamérica. Revista Semestral Enero-Junio 2017

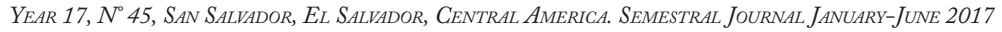

\title{
La importancia de la contabilidad electrónica en el currículum
}

\section{The importance of electronic accounting in the curriculum}

\author{
Mtro. Pedro Lee Pérez \\ Contador Público, Maestría en Fiscal y Doctorando en \\ Ciencias del Desarrollo Humano y Organizacional \\ Universidad del Valle de Atemajac, Plantel Guadalajara \\ pedro.lee@univa.mx \\ Fecha de recepción: 25 de febrero de 2017 \\ Fecha de aceptación: 4 de mayo de 2017
}

\section{RESUMEN}

El presente artículo habla sobre la contabilidad electrónica en el sistema tributario de México. Esto es importante para los contribuyentes a partir de 2014, porque obliga a las personas físicas y morales del país a llevar el registro de la contabilidad en medios electrónicos y su envío periódico a través del buzón tributario del Servicio de Administración Tributaria (SAT). El objetivo de este artículo es argumentar la necesidad de incluir la contabilidad electrónica en el currículum de la Licenciatura en Contaduría Pública, al considerar que los perfiles de egreso de la mayoría de las universidades, aún siguen el desarrollo de las competencias tecnológicas que exigen hoy en día las obligaciones tributarias.

Palabras clave: obligación tributaria, México, SAT, Hacienda Federal, fisco.

\section{ABSTRACT}

This article discusses electronic accounting in the Mexican tax system, this is important for taxpayers as of 2014, because it obligates the physical and moral persons of the country to keep records of accounting in electronic media and its sending Newspaper through the tax mailbox of the Tax Administration Service (SAT). The objective of this article is to argue the need to include electronic accounting in the curriculum of the Degree in Public Accounting, considering that the exit profiles of most universities, still follow the development of the technological skills that demand today in day tax obligations.

Keywords: tax obligation, Mexico, SAT, Federal Treasury, treasury. 


\section{Introducción}

Antes de destacar la inclusión de la técnica de la contabilidad electrónica en un plan de estudios del contador público, se considera importante definir desde el punto de vista conceptual cómo debemos de entender la contabilidad electrónica. Ya que en el medio académico no se ha encontrado una definición de algún autor que nos resulte convincente y suficientemente completa para el presente artículo, la aproximación a la interpretación del concepto se plantea desde la autoridad fiscal del país, tomando en cuenta el momento en el cual decidió apoyarse en la tecnología para aplicarla como base en la generación y envío de la disposición que denominó como Contabilidad Electrónica.

Se señala que la Contabilidad Electrónica se trata de una técnica que involucra e interrelaciona tres áreas de conocimiento que son: la contable, la jurídica y la tecnológica. Estos elementos deberán de trabajarse de manera integral para lograr una implementación del esquema tributario, acatando de manera correcta la exigencia fiscal, la cual deben cumplir las personas físicas $y$ morales que están obligadas a llevar contabilidad por medios electrónicos, tanto para su envío y registro, así como para toda la documentación e información relacionada con el cumplimiento de las disposiciones fiscales.

Para generar la información contable y hacer el envío al Servicio de Administración Tributaria (SAT), se demanda de un mayor esfuerzo y bagaje de conocimientos del profesional en Contaduría Pública en lo que respecta al área tecnológica, puesto que en la formación académica de su carrera suele carecerse de una asignatura que lo capacite para operar la contabilidad electrónica, teniendo que recurrir a talleres y cursos de capacitación impartidos por las empresas productoras de software, asesores o colegios de contadores.

En el contexto mundial, de acuerdo a la tendencia y evolución del uso de servicios electrónicos para la prestación de servicios al contribuyente, en el informe de la encuesta del Foro sobre la Administración Tributaria, se destaca que a nivel mundial se ha tenido un progreso general a alto nivel desde 2004, en los servicios basados en internet, también han tenido los países un progreso general considerable en el uso de la declaración electrónica de los principales tipos de impuestos; sin embargo, cerca del 30\% de los países encuestados miembros de la OCDE, siguen teniendo pendiente grandes avances en algunos o todos los impuestos primordiales (OCDE, 2009). Así mismo, la mayoría de los organismos tributarios están en el proceso de transformación de la madurez general de sus servicios electrónicos, teniendo todavía camino que recorrer para lograr el nivel que establece el modelo de madurez del gobierno electrónico de la OCDE.

Hoy en día, México se encuentra en ese proceso de transformación de los servicios electrónicos y se puede destacar que la autoridad fiscal cuenta con un portal a través del cual se hace la mayoría de trámites de los contribuyentes vía internet. De manera adicional, los causantes hacen declaraciones de impuestos electrónicos y pago de impuestos electrónicos, reciben acuses electrónicos, expiden y reciben facturas electrónicas y elaboran la nómina en forma digital (SAT, 2015). La contabilidad electrónica 
es un paso más en ese proceso de transformación del cumplimiento tributario hacia la modalidad en línea.

En México, en los últimos años, las empresas tienen una nueva obligación fiscal del envío de la contabilidad electrónica al SAT, "Ingresarán de forma mensual su información contable a través de la página de Internet del Servicio de Administración Tributaria, de conformidad con reglas de carácter general que se emitan para tal efecto" (Martínez, 2016, pág. 19), para la cual no estaban preparados las entidades económicas, los profesionales de la contaduría y la sociedad en general, representando una carga administrativa adicional para las empresas y una demanda de esfuerzo y recursos para la capacitación en la nueva tecnología para los contadores. Esta situación originó una preocupación general entre los ciudadanos, por tener la percepción de una fiscalización permanente a las personas físicas y morales de todos sus actos o actividades empresariales, por el envío periódico al SAT de la información contable con el siguiente procedimiento: "Contar con la firma electrónica activa y vigente. Contar con el (los) archivo(s) XML comprimidos en formato .ZIP con la nomenclatura establecida. Contar con acceso a internet para realizar el envió" (Chamlaty, 2016, pág. 124).

A partir de 2014, se estableció la obligación de registrar la información contable en: "medios electrónicos que es toda aquella herramienta, programa, aplicación o dispositivo que nos permita representar o almacenar la información en un formato que un equipo o sistema de cómputo puedan procesar" (Flores, 2016, pág. 22) y aunque una importante cantidad de contribuyentes llevan desde hacía tiempo su contabilidad en sistemas y estaban familiarizados con el uso de la tecnología, no estaban aún preparados para presentar sus archivos contables bajo parámetros uniformes y homologados, ni para transformar esos registros en formatos estandarizados de carácter digital, condiciones requeridas para su presentación al SAT; por ello el cumplimiento de la obligación se pospuso al ejercicio de 2015, sólo para algunos contribuyentes con mayor capacidad administrativa, y para 2016, el resto.

Lo anterior es lo que motiva el objetivo del presente artículo, el cual es argumentar la necesidad de incluir la contabilidad electrónica en el currículum de la Licenciatura en Contaduría Pública, al considerar que los perfiles de egreso de la mayoría de las universidades, aún siguen el desarrollo de las competencias tecnológicas que exigen hoy en día las obligaciones tributarias.

\section{Desarrollo}

\section{E1 nuevo currículum en la sociedad de la información}

Con el fin de proponer la asignatura de Contabilidad Electrónica para que se incluya en el plan de estudios para el mejoramiento del proceso de la construcción del profesional de la Contaduría Pública, se considera necesario indagar los temas de la teoría curricular, la sociedad de la información y los nuevos campos profesionales, el currículum y el diseño curricular, los cuales serán la base de la presente reflexión.

La Teoría Curricular nace como expresión de una "nueva" articulación entre escuelasociedad. La mediación de esta articulación está proporcionada por la génesis de la 
industrialización a través de máquinas y con el surgimiento de los grandes monopolios (Ibarra, La teoría curricular, 2013, pág. 23).

La formación académica nos remite a ese apartado del currículum que describe la trayectoria formativa del profesional, la cual refleja una línea temporal a lo largo de la que la persona ha podido cursar unos estudios determinados.

En la actualidad, la gestión del conocimiento se hace de diferentes maneras y formas, cambiando constantemente y provocando la transición de economías industriales a economías y sociedades basadas en el conocimiento y transformando la naturaleza de la educación, del trabajo, de la producción y de la cultura (UNESCO, 2005).

Según Esteinou (2003), la visión de la sociedad civil concibe a las sociedades de información y de la comunicación como realidades basadas en los derechos humanos y en el desarrollo humano duradero, así como también que ese desarrollo esté centrado en la satisfacción de necesidades humanas fundamentales $y$ en claros objetivos sociales, culturales, económicos y de medioambiente. Estos mismos deben dar prioridad al abatimiento de la pobreza y el apoyo también a sociedades donde el conocimiento, los valores, la comunicación y la organización sean consideradas como factores de importancia, y donde la persona tenga la oportunidad no solo de acceder a la información sino de producirla y ser creativa e innovadora, basado en la cooperación y trabajo en equipo.

Actualmente, el uso de las redes ha adquirido un incremento importante y considerable de tal modo, que: "la interacción focalizada en las redes, no debe entenderse solamente como una interconexión tecnológica, sino como la interconexión de individuos valiéndose del uso de la tecnología disponible; posibilitando justamente que por medio de redes, se pueda combinar conocimiento, creatividad e innovación, para avanzar en la consecución de un desarrollo social sustentable y equitativo" (Ibarra, La gestión del conocimiento en la sociedad del conocimiento, 2013, pág. 265).

Esta forma de comunicación de la era digital está cambiando constantemente la manera de gestionar el conocimiento y ponerlo a circular en la sociedad, pero desde el punto de vista conceptual cómo se define a la sociedad de la información.

Aldana (2000) señala que para ManuelCastellsésta es como un nuevo sistema tecnológico, económico y social, una economía en la que el incremento de productividad no depende del incremento cuantitativo de los factores de producción (capital, trabajo, recursos naturales), sino de la aplicación de conocimientos e información a la gestión, producción y distribución, tanto en los procesos como en los productos.

$\mathrm{Y}$ en relación con el sistema tecnológico de la anterior definición, podemos agregar la recomendación de la Organización de las Naciones Unidas para la Educación, la Ciencia y la Cultura (UNESCO) "difundir las nuevas tecnologías llamadas sociedades de la información en favor de todos los países, a fin de evitar una agudización aun mayor de las diferencias entre países ricos y pobres” (Delors, 1996, pág. 29).

De igual manera, también un importante número de países ha puesto en marcha en los 
últimos años iniciativas para el desarrollo o simplemente la promoción de la Sociedad de la Información. En sus títulos suele introducirse la expresión Sociedad de la Información. Son los casos de Reino Unido (Iniciativa de La Sociedad de la Información), Francia (Programa de la Acción Gubernamental por la Sociedad de la Información), Finlandia (camino de Finlandia a la Sociedad de la Información), España (Info XXI, la Sociedad de la Información para todos) o incluso Andorra (Plan de Acciones para facilitar la transición de Andorra a una Sociedad de la Información).

La sociedad de la información se sitúa como un factor de cambio, que transforma en un proceso lento pero progresivo a una sociedad inspirada en el conocimiento y que argumenta, cuestiona e incide en la sociedad, aplicando nuevos sistemas y generando nuevos campos en todas las ramas de las profesiones.

Los nuevos campos profesionales se empezaron a generar a nivel de técnicos, ya que debido al uso de las computadoras y la descompostura de una de sus partes, que en la mayoría de los casos fue el disco duro, ocasionó que algunas personas (técnicos) se encargaran de dar el mantenimiento y la reparación correspondiente de la computadora dando como consecuencia que se generara el primer nuevo campo profesional, y posteriormente aparecieron los técnicos operadores y capturistas, así como los técnicos programadores y analistas.

En cuanto al nuevo campo profesional nivel Licenciatura, se generó en el año de 1982, con el programa CAD, para diseño asistido para dibujo y modelado en segundo y tercera dimensión
(2D y 3D), para arquitectos e ingenieros y posteriormente en el año de 1985, se generó un nuevo campo profesional a nivel especialidad, con la primera especialidad denominada: Informática Médica, y posteriormente en el año de 1999, se generó un nuevo campo profesional con el programa COTTAGEMED, para médicos, que tenía como base informativa las historias clínicas de los pacientes.

En la década de los ochenta, surgieron los programas: Procesador de texto (Word), Hoja de cálculo (Excel), generando nuevos campos en todas las profesiones, entre ellas la de Contaduría Pública y posteriormente a mediados de la década de los 90 , surgieron en nuestro país, los primeros programas o software de contabilidad para el registro de las operaciones de las empresas. A partir de 2014, con la obligación fiscal del envío de la contabilidad electrónica, se empezaron a generar nuevos campos dentro de la profesión de la contaduría.

La formación académica de una profesión nos remite a ese apartado del currículum que describe la trayectoria formativa del profesional, la cual refleja una línea temporal a lo largo de la cual una persona ha podido cursar estudios determinados.

Currículo proviene del currículum, de curraré, "correr", que significa "carrera”. En sus orígenes el término currículo se entendía en un sentido algo más restringido, pues venía asociado a lo que debía enseñarse en las escuelas, haciendo referencia exclusiva a los contenidos de las disciplinas y al plan de estudios de una determinada materia.

Ibarra (2010) indica que el concepto de currículum "es creado en el contexto de una 
pedagogía de la sociedad industrial, aunque se ha hecho uso de él, en extensiones arbitrarias como organizador de contenidos temáticos casi neutros en circunstancias históricas diversas a las de su origen”.

Mediante la construcción curricular, la institución plasma su forma de concebir y organizar el proceso educativo, de esta manera, el currículo permite la predeterminación de la construcción de la formación académica del ser, para poder lograr el modelo de individuo que se pretende generar a través de la implementación del mismo.

Un modelo de currículo es capaz de servir por sí solo a una escuela que respeta las diferencias, realizando transformaciones para atender a las necesidades de los diferentes alumnos. Algunos principios fundamentales del currículo: ser relevante en los contenidos; flexible a los cambios en las necesidades de los alumnos y en las técnicas empleadas por los profesores; tener una base amplia en cuanto a la comunicación y participación de los profesores, padres, administradores y comunidad en general; ser realista en sus propuestas $y$ racional en su desarrollo y sobre todo observar las diferencias individuales en el aprendizaje, no como algo estático, sino dinámico, interactivo y en continua evolución.

Franklin Bobbit, en el primer trabajo formal sobre el tema, inmerso en la corriente funcionalista, consideró dos acepciones de currículum: como experiencias dirigidas al logro de habilidades conscientes o no, y la otra como experiencias premeditadas para dicho fin, en una escuela cuyo fin era servir y reflejar el modelo industrial, tomando como referencia la administración de
Taylor. El currículum para Bobbit aparece como una descripción de objetivos a lograr, a través de procedimientos a medida. Para Taylor, el currículum debe dar respuestas a los fines, a los logros de experiencias, a los modos de alcanzar esos fines y las maneras de comprobarlo.

\section{La necesidad de un rediseño curricular}

El diseño curricular, como proceso de elaboración de un currículo de estudio, debe estar fundamentado en determinado presupuesto teórico y metodológico. El modelo curricular tecnológico es el enfoque que ha caracterizado y determinado la mayoría de los modelos funcionales de diseño curricular que han surgido desde principios del siglo $\mathrm{XX}$ hasta nuestros días. El mismo se sustenta en la tecnología de la educación entendida como el desarrollo de un conjunto de técnicas sistemáticas, junto a conocimientos prácticos, para el diseño, la validación y la operacionalización de las escuelas como sistemas educativos.

En México, el campo del currículo se ha denominado, siguiendo la concepción de desarrollo curricular, ya que "la noción de desarrollo abarca una multiplicidad de procesos, estructuras, prácticas educativas, relacionadas con los proyectos curriculares y su concreción" (Díaz Barriga Arceo, 2005, pág. 5).

"En el enfoque desarrollo curricular, el currículum renace como una estrategia que planifica, organiza, sustenta y genera la acción educativa, como un paradigma que conjuga planteamientos administrativos y económico-financieros a la ciencia educativa. Por lo tanto, el quehacer educativo institucional se comprende como el 
proceso de desarrollo curricular; este constituye en su conjunto, un sistema general que rige y orienta las funciones de la universidad hacia el trabajo educativo" (Ibarra, 2004, p.196).

En el desarrollo del currículum en la formación académica de la contabilidad electrónica, deberá de hacerse de acuerdo con notas sobre planes de estudio y relaciones disciplinares en el currículo. "Para desarrollar el plan educativo de cada una de las profesiones que ofrece la institución se hace necesario el proceso de diseño curricular con el objeto de estructurar los conocimientos y experiencias de aprendizaje que en forma intencional, se articulan con la finalidad de producir enseñanzas y aprendizajes que a su vez se traduzcan en formas de pensar y actuar ante los problemas concretos que plantea la vida social y la incorporación al trabajo" (Pansza, 1987, pág. 36).

\section{Discusión argumentada}

La iniciativa del Decreto de la Reforma Fiscal 2014 planteó la necesidad de "definir condiciones para que los contribuyentes cumplan con elementos mínimos y características específicas y uniformes, además de que la autoridad fiscal cuente con una contabilidad estructurada”.

También como parte de los cambios de la citada reforma, con relación a la modernización del cumplimiento de las obligaciones fiscales se consideró establecer un esquema innovador y práctico, que retomara los beneficios y experiencias de la utilización de medios electrónicos y estándares tecnológicos abiertos para implantar, respecto a la obligación de los contribuyentes de llevar contabilidad: que ésta sea electrónica y que la misma se genere en tres tipos de reportes electrónicos a entregar a la autoridad fiscal (Servicio de Administracion Tributaria, 2015, pág. 36).

Con base en estos planteamientos de la autoridad fiscal de México, nos permitimos inferir que el currículum de la Licenciatura en Contaduría Pública, contemple la Contabilidad Electrónica, como parte de la formación académica al considerar que los perfiles de egreso de la mayoría de las universidades, aún siguen el desarrollo de las competencias tecnológicas que exigen hoy en día las obligaciones tributarias.

Díaz (2012) propone que las tareas o acciones de la práctica profesional, se definen a partir de tres elementos, los cuales son:

1. Las necesidades sociales detectadas, a las cuales tratará de dar solución el profesionista.

\section{El mercado ocupacional.}

3. El análisis de las disciplinas que puedan aportar elementos para la solución de dichos problemas.

Con sustento en los anteriores elementos, podemos tomar en consideración la disciplina de la Contabilidad Electrónica en el rediseño del currículum de Licenciado Contador Público, en virtud de que un profesionista soluciona la necesidad social del cumplimiento de la obligación fiscal.

Además, en el mercado laboral existen despachos de contadores y personas morales y físicas con actividad empresarial, que requieren profesionistas para cumplir sus obligaciones tributarias. 
Finalmente, el análisis de la disciplina de la Contabilidad Electrónica es la que soluciona una necesidad social.

Por tal motivo es importante, como la misma autora señala, la necesidad de preparar los recursos humanos que puedan enfrentarse a esos problemas y las características académicas y laborales que debe poseer la persona que tratará de satisfacer dichas necesidades.

Y además, define el perfil profesional como: "la determinación de las acciones generales y específicas que desarrolla un profesional en las áreas o campos de acción (emanados de la realidad social y de la propia disciplina) tendientes a la solución de las necesidades sociales previamente advertidas".

Con base en la necesidad de preparar los recursos humanos y la definición de la autora de referencia, podemos estimar conveniente el proyecto de rediseño del currículum para la formación académica de los estudiantes de la carrera de Contaduría en las universidades, para satisfacer las necesidades de la sociedad actual.

Respecto a la valoración externa del currículo se dice que: "se refiere principalmente al impacto social que puede tener el egresado" (Díaz, 2010).

Por lo tanto, una valoración externa aceptable del plan de estudios de Licenciatura en Contaduría Pública demanda que el egresado posea conocimientos de la Contabilidad Electrónica, para el desempeño profesional eficaz, eficiente y que impacte en un ambiente donde se transforma la sociedad industrial y se reclama un cambio para responder a las necesidades de la era digital.

\section{Cierre}

Las obligaciones tributarias cumplimentadas por vía electrónica, por parte de los profesionales de la Contaduría, es un tema de gran relevancia hoy en día, en las universidades. Debemos considerar la necesidad de la inclusión de la disciplina de la Contabilidad Electrónica en el plan de estudios de la Licenciatura de Contaduría Pública, con la finalidad de que los egresados, posean las competencias contables, fiscales y tecnológicas para el registro de operaciones y envío periódico de la información contable de las personas morales y físicas, al Servicio de Administración Tributaria.

Si bien no todos los planes de estudios de la carrera de Contaduría contemplan la Contabilidad Electrónica, los académicos de las universidades deben afrontar la responsabilidad de su revisión y actualización, con el propósito de abonar a la evaluación externa del currículum, por la sociedad.

Ahora, para la puesta en práctica estimamos conveniente que los docentes de Contaduría de las universidades deberán contar con las capacidades siguientes: conocer métodos didácticos específicos de la Contabilidad Electrónica, la competencia tecnológica práctica de la técnica y discernir el acompañamiento adecuado a los alumnos en el proceso formativo de la Contabilidad, en medios electrónicos.

La Contabilidad Electrónica es significativa para la sociedad en general, ya que se trata de una norma jurídica y el incumplimiento de ella por parte de los contribuyentes trae consigo molestias administrativas y sanciones económicas. 


\section{Referencias bibliográficas}

Aldana, M. (2000). Reseña de "La era de la información, realidades y reflexiones sobre la globalización" de Manuel Castells. Recuperado en septiembre de 2016, de Espiral, vol. VI, núm. 18, pp. 285-316: http://www.redalyc.org/comocitar. oa?id=13861811

Chamlaty, M. (2016). Análisis de la Contabilidad Electrónica. México: Gasca.

Delors, J. (1996). La educación encierra un tesoro. Francia: UNESCO.

Díaz Barriga Arceo, F. (2005). Desarrollo del currículo e innovación:Modelos e investigación en los noventa. Recuperado en septiembre de 2016, de Perfiles Educativos vol. XXVII núm. 107, pp. 57-84: http://www.scielo.org.mx/pdf/ peredu/v27n107/n107a04.pdf

Díaz, F. (2012). Metodología de Diseño Curricular para la Educación Superior. Mexico: Trillas.

Esteinou, J. (2003). Hacia una nueva sociedad de la comunicacion y de la informacion. Razon y Palabra.

7Flores, J. (2016). Contabilidad Electrónica en 5 pasos. México: Taxxx.
Ibarra, A. (2013). La gestión del conocimiento en la sociedad del conocimiento. Universidad del Valle de Atemajac, 265.

Ibarra, A. (2013). La teoría curricular. Universidad del Valle de Atemajac, 23.

Martínez, J. (2016). Estudio Práctico sobre la Balanza de Comprobación para efectos del SAT. México: ISEF.

OCDE. (2009). OECD.ORG. Recuperado el 18 de diciembre de 2016, de http://www.oecd. org/ctp/administration/46668703.pdf

Pansza, M. (1987). Notas sobre planes de estudio y relaciones disciplinares en el currículo. Perfiles educativos, 36.

Servicio de Administracion Tributaria. (2015). La Nueva Administración Tributaria en México 2a Edición. México: Amexipac.

UNESCO. (2005). Informe Mundial de la UNESCO. Hacia las sociedades del conocimiento. Recuperado el 17 de febrero de 2017, de Ediciones UNESCO: http://unesdoc.unesco. org/images/0014/001419/141908s.pdf 